\title{
Preface to Volume 2019, Issue 1
}

\author{
Florian Mendel ${ }^{1}$ and Yu Sasaki ${ }^{2}$ \\ ${ }^{1}$ Infineon Technologies, Neubiberg, Germany \\ florian.mendel@gmail.com \\ ${ }^{2}$ NTT Secure Platform Laboratories, Tokyo, Japan \\ yu.sasaki.sk@hco.ntt.co.jp
}

IACR Transactions on Symmetric Cryptology (ToSC) is a forum for original results in all areas of symmetric cryptography, including the design and analysis of block ciphers, stream ciphers, encryption schemes, hash functions, message authentication codes, (cryptographic) permutations, authenticated encryption schemes, cryptanalysis and evaluation tools, and security issues and solutions regarding their implementation.

ToSC implements an open-access journal/conference hybrid model following some other communities in computer science. All articles undergo a journal-style reviewing process and accepted papers are published in gold open access (in our case the Creative Commons License CC-BY 4.0). The review procedures that we have followed strictly adhere to the traditions of the journal world. Full papers are assigned to the members of the Editorial Board. These members write detailed and careful reviews (usually without relying on subreviewers). Moreover, we have had a rebuttal phase, allowing authors to respond to the review comments before the final decisions. If necessary, the review process enables further interactions between the authors and the reviewers, mediated by the Co-Editors-in-Chief. Detailed discussions among the reviewers lead to one of the following four decisions for each paper: ACCEPT, in which case the authors submit their final camera-ready manuscript after editorial corrections; ACCEPT with MINOR REVISION, which means that the authors revise their manuscript and go through one or more iterations and reviews of the manuscript until the comments have been addressed in a satisfactory way; MAJOR REVISION, which means that the authors are requested to make major changes to their manuscript before submitting again in one of the next rounds; and REJECT, which means that the manuscript is deemed to be not suitable for publication in ToSC. The last four issues we have tried to refine the method (new for a community used to only accept or reject decisions) and decide in a more fair way when to assign major revisions.

The review process shares with the high quality conferences that it is double-blind and adheres to a strict timing; but unlike a traditional conference, there are multiple submission deadlines per year. Each paper received at least three reviews; for submissions by Editorial Board members this was increased to at least four.

Overall, we are very pleased with the quality and quantity of submissions, the detailed review reports written by the reviewers and the substantial efforts by the authors to further improve the quality of their work. We think that the review process leads to an increased quality of the papers that are published.

The papers selected by the Editorial Board for publication in the last four issues were presented at the conference Fast Software Encryption (FSE). This gave the authors the opportunity to advertise their results and engage in discussions on further work. In 2019, FSE was held during March 25-28, 2019 in Paris, France. The papers presented at FSE 2019 appeared in ToSC Volume 2018, Issues 2-4 and Volume 2019, Issue 1. For Volume 2018, Issue 2, we received 25 submissions, out of which 7 were accepted, 2 of these after minor revisions; the number of papers that received a major revision decision was 5 . 
For Volume 2018, Issue 3, we received 31 submissions, out of which 10 were accepted, 4 of these after minor revisions; the number of papers that received a major revision decision was 3. For Volume 2018, Issue 4, we received 41 submissions, out of which 8 were accepted, 5 of these after minor revisions; the number of papers that received a major revision decision was 5. For Volume 2019, Issue 1, we received 45 submissions, out of which 11 were accepted, 3 of these after minor revisions; the number of papers that received a major revision decision was 8 .

Besides the 36 selected talks, the program included one invited talk by Gregor Leander on non-linear invariant attacks, María Naya-Plasencia on post-quantum security of symmetric-key cryptography, and Jian Guo on cryptanalysis of Keccak based constructions. The conference also featured a rump session, chaired by Pierre Karpman and Brice Minaud, with several short informal presentations. As it is tradition for FSE, the Editorial Board also selected a best paper, based on the scientific quality and contribution. The Editorial Board has decided to give the award to the paper by Léo Perrin entitled "Partitions in the S-Box of Streebog and Kuznyechik".

We would like to thank the authors of all submissions for contributing high quality submissions and giving us the opportunity to compile a good and diverse program. In particular, we would like to thank the Editorial Board members; we value their hard work and dedication to write constructive and detailed reviews and to engage in interesting discussions. Many Editorial Board members spent additional time as shepherds to help the authors improving their works. We would also like to thank the subreviewers for their efforts. We are profoundly indebted to the conference General Chair Jérémy Jean for his hard work to make the conference a success. We also would like to thank Anne Canteaut, Shai Halevi, Gregor Leander, and Friedrich Wiemer for their work and support. Finally, we would like to thank ANSSI, Thalès, INRIA, Ledger, ENS PSL, Pôle d'Excellence Cyber, CryptoExperts, Idemia, CyberCrypt, DIM RFSI, and Région Ile de France for their generous support of the conference.

We hope that the papers in this volume of IACR Transactions on Symmetric Cryptology (ToSC) prove valuable and we are glad to see that ToSC is becoming the leading international venue publishing the top research on symmetric cryptology.

March 2019

Florian Mendel

Yu Sasaki 


\section{Editorial Board}

Frederik Armknecht Subhadeep Banik

Daniel J. Bernstein

Alex Biryukov

Christina Boura

Anne Canteaut

Carlos Cid

Joan Daemen

Patrick Derbez

Itai Dinur

Christoph Dobraunig

Orr Dunkelman

Maria Eichlseder

Jian Guo

Takanori Isobe

Tetsu Iwata

Jérémy Jean

Pierre Karpman

Nathan Keller

Stefan Kölbl

Virginie Lallemand

Gregor Leander

Jooyoung Lee

Gaëtan Leurent

Stefan Lucks

Subhamoy Maitra

Willi Meier

Bart Mennink

Nicky Mouha

Maria Naya-Plasencia

Samuel Neves

Ivica Nikolić

Kaisa Nyberg

Bart Preneel

Arnab Roy

Martin Schläffer

Yannick Seurin

Hadi Soleimany

Ling Song

Marc Stevens

Elmar Tischhauser

Yosuke Todo

Gilles Van Assche

Damian Vizár

Lei Wang
University of Mannheim, Germany

EPFL, Switzerland

University of Illinois at Chicago, USA

University of Luxembourg, Luxembourg

University of Versailles, France

Inria, France

Royal Holloway University of London, United

Kingdom

Radboud University, Netherlands and STMi-

croelectronics Belgium

University of Rennes 1, France

Ben Gurion University, Israel

TU Graz, Austria

University of Haifa, Israel

TU Graz, Austria

NTU, Singapore

University of Hyogo, Japan

Nagoya University, Japan

ANSSI, France

Université Grenoble Alpes, France

Bar-Ilan University, Israel

DTU, Denmark

University of Bochum, Germany

University of Bochum, Germany

KAIST, Korea

Inria, France

Bauhaus-Universität Weimar, Germany

ISI, India

FHNW, Switzerland

Radboud University, Netherlands

NIST, USA

Inria, France

University of Coimbra, Portugal

National University of Singapore, Singapore

Aalto University, Finland

KU Leuven, Belgium

University of Bristol, United Kingdom

Infineon Technologies, Germany

ANSSI, France

Shahid Beheshti University, Iran

NTU, Singapore and Chinese Academy of Sci-

ences, China

CWI, Netherlands

DTU, Denmark

NTT Secure Platform Laboratories, Japan

STMicroelectronics, Belgium

EPFL, Switzerland

Shanghai Jiao Tong University, China 


\section{External reviewers}

Christof Beierle

Lauren De Meyer

Johann Großschädl

Vasily Mikhalev

Kazuhiko Minematsu

Francois-Xavier Standaert

Aleksei Udovenko

Qingju Wang 